\title{
A case report of colouterine fistula due to colonic diverticulitis: a rare complication
}

\author{
Shweta Pandey ${ }^{1}$, Saurabh Maheshwari ${ }^{{ }^{*}} \mathbb{D}$, Uddandam Rajesh ${ }^{1}$, Darshan Singh Grewal ${ }^{1}$ and Vibhuti Maria ${ }^{2}$
}

\begin{abstract}
Background: Colouterine fistula is a very rare entity because of the sturdy and muscular nature of the uterus. Due to the rarity of this condition and nonspecific clinical findings, it poses a diagnostic challenge to treating physicians.

Case presentation: Here, we report a case of a 64-year-old female, who presented with lower abdominal pain. Clinically, there was suspicion of a recto-vaginal fistula. However, on imaging, she was found to have a colouterine fistula secondary to sigmoid diverticulitis.

Conclusion: It is essential to be aware of this rare complication of sigmoid diverticulitis. The imaging plays a cardinal role in the diagnosis and guiding the management of this disease.
\end{abstract}

Keywords: Diverticulosis, Diverticulitis, Colouterine, Fistula, Complication

\section{Background}

Diverticular disease of the colon is a commonly seen entity in the developed countries. The prevalence of this disease among Asian countries is comparatively quite low. Consumption of a diet having a low fiber content has been attributed to have a major role in the development of diverticular disease. There has been an increase in its incidence in Asian countries due to the adoption of western dietary habits. The incidence of colonic diverticulosis also increases with aging [1].

Diverticulitis due to infection and inflammation of the diverticula is a common complication of diverticular diseases with a complication rate of around 20\%. Diverticulitis itself can result in various secondary complications like stricture formation, perforation, bleeding, and formation of a fistula. Fistula formation, which is communication between two surfaces lined by epithelium, accounts for $\sim 2 \%$ of total cases of complicated diverticulitis [1].

The thick musculature of the uterus is generally resistant to involvement by benign or malignant disease.

\footnotetext{
* Correspondence: saurabhmhshwr@yahoo.co.in

${ }^{1}$ Department of Radiodiagnosis and Imaging, Armed Forces Medical College, Pune, India

Full list of author information is available at the end of the article
}

Hence, a colouterine fistula is a rare occurrence, particularly as a complication of diverticulitis [2]. We report a case of sigmoid diverticulitis with the formation of colouterine fistula in a 64-year-old female.

\section{Case presentation}

A 64-year-old female presented with complaints of lower abdominal pain in the region of left iliac fossa and supra-pubic region of 1-month duration. She was evaluated for urinary tract infection, and a gynecological consult was also taken. On per speculum examination, fecal matter was seen in the vagina and a provisional diagnosis of the rectovaginal fistula was made. She did not have prior history of similar symptoms. Her medical history and family history were unremarkable.

A contrast-enhanced computed tomography was performed on a 16 slice CT scanner. Positive oral and rectal iodinated contrast was administered followed by intravenous administration of $60 \mathrm{ml}$ of non-ioniciodinated water-soluble contrast at a rate of $2.5 \mathrm{ml} / \mathrm{s}$. CECT images of the pelvis showed multiple small outpouchings arising from the sigmoid colon with contrast filled in them, which was consistent with diverticulosis. An air- and contrast-filled outpouching was seen arising from the anterior wall of the sigmoid colon and 
projecting between the urinary bladder and uterus. There was uniform wall thickening of this segment of bowel with surrounding fat stranding and heterogeneous post-contrast enhancement (Fig. 1). Contrast along with air locules was noted in the endometrial cavity and cervix. However, a definite fistulous track was not well delineated on CT images. There was thickening of the subjacent posterior wall of the urinary bladder. No contrast was seen in the lumen of the urinary bladder.

Magnetic resonance imaging (MRI) of the pelvis was performed with the acquisition of multiplanar T1weighted images (T1WI), T2-weighted images (T2WI), diffusion-weighted images (DWI), and dynamic postgadolinium T1-weighted fat-saturated images. The findings seen on CECT were confirmed, and the fistulous tract between the inflamed diverticula and the uterine cavity was well delineated (Fig. 2). Hence, a final diagnosis of colouterine fistula secondary to sigmoid diverticulitis was made.

Our patient is planned for management in two stages. In the first stage, an end-colostomy has been created to allow healing of inflamed diverticulum. The second stage of resection and anastomosis will be performed in the future. Presently, patient is asymptomatic and tolerating the end-colostomy well.

\section{Ethics approval and consent to participate}

The present study was approved by the ethical board of the hospital in which the study was performed. The patient reported in this article had signed a written informed consent form. This case report was a reporting of a case in a medical educational center, in which all patients are informed that they may be subjects of scientific experiments and are informed of the ethical codes of conducts. This study was in compliance to the latest version of the Helsinki Declaration.

\section{Discussion}

Diverticular diseases of the colon are prevalent in Western countries and are one of the leading causes for outpatient visits and hospitalizations [3]. Its prevalence shows wide geographic and ethnic variability. It is considered to be low in the Asian population, mostly due to the higher fiber content in the diet [4].

Diverticulitis is inflammation or infection in diverticula and is one of the most common complications of diverticulosis. Approximately $20 \%$ of patients with colonic diverticula develop this complication. Diverticulitis can further complicate to give rise to conditions like obstruction, hemorrhage, abscess, and fistula formation. Fistula formation is a relatively rare complication of diverticulitis. It accounts for approximately $20 \%$ of surgeries for complicated diverticulitis [4].
Among fistulous complications of diverticulitis, a colovesical fistula is the most common type, followed by a colovaginal fistula. As the uterus is a thick and muscular organ, it resists invasion and the spread of inflammation. Hence, colovesical fistula is also a more common occurrence in males as compared to females. The females who have undergone hysterectomy show an equal incidence of colovesical fistula as in men, due to loss of this protective barrier [5].

The first case of colouterine fistula was reported by Lejemtel in 1909 [6]. According to his paper, the main causative etiologies identified for this condition were uterine trauma, rupture of abscess into the bowel, and carcinoma of the uterus and sigmoid colon. Later studies added radiotherapy as one of the etiological factors. Iatrogenic causes like invasive procedures and surgeries can also lead to this condition. Infectious causes remain the key etiological factors. Infection of the bowel can lead to severe inflammation and abscess formation which can decompress by perforating into subjacent pelvic organs like the uterus, vagina, and urinary bladder or through the body surface [5].

The fistulous communication between the colon and the urinary bladder (colovesical fistula) can be seen in up to $65 \%$ of the cases whereas between the colon and the vagina (colovaginal) in about $25 \%$ of the patients. Colouterine fistula is a rare complication of diverticulitis of the colon. Clinical presentation of the colouterine fistula can be varied. As the colon communicates with the uterus through a fistula tract, these patients generally present with foul-smelling fecal or purulent vaginal discharge [7].

Various radiological imaging modalities have been used for diagnosing colouterine fistula. Ultrasound is a commonly available, inexpensive, and safe imaging modality for gynecologic diseases. It is a common first-line imaging modality that is used to analyze the uterine cavity and endometrium. Although it has limited diagnostic value in the diagnosis of colouterine fistula, it plays a major role in guiding further diagnostic work-up in these patients. Sonohysterography with contrast medium can be used to demonstrate the fistula track for diagnosis of colouterine fistula [8].

There has been an increase in the use of computed tomography nowadays in the setting of an acute abdomen, and it has also been used increasingly in the evaluation of fistulous diseases of the abdomen and pelvis. In colouterine fistula, computed tomography can demonstrate the joining of walls of the uterine cavity and colon. The presence of air bubbles in the uterine cavity further strengthens the diagnosis. However, failure of the demonstration of the exact fistulous tract in all cases remains a primary limitation of this modality. Multiplanar reconstructions on multidetector $\mathrm{CT}$ allows improved 


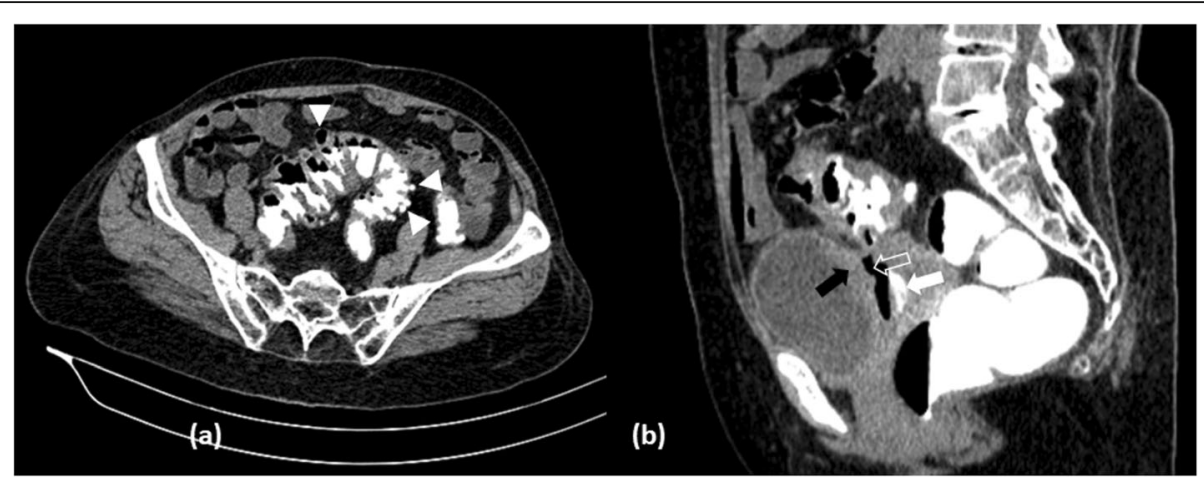

Fig. 1 Contrast-enhanced computed tomography (CT) images of the pelvis: axial image (a) in soft tissue window shows multiple contrast and airfilled diverticula arising from the sigmoid colon (solid white arrowheads). Sagittal reformat image (b) shows presence of an air-filled outpouching of sigmoid colon in the utero-vesical space (void white arrow). The rectally administered positive contrast media is seen to reach within the endometrial cavity (solid white arrow). Note is also made of thickening of posterior wall of urinary bladder (solid black arrow)

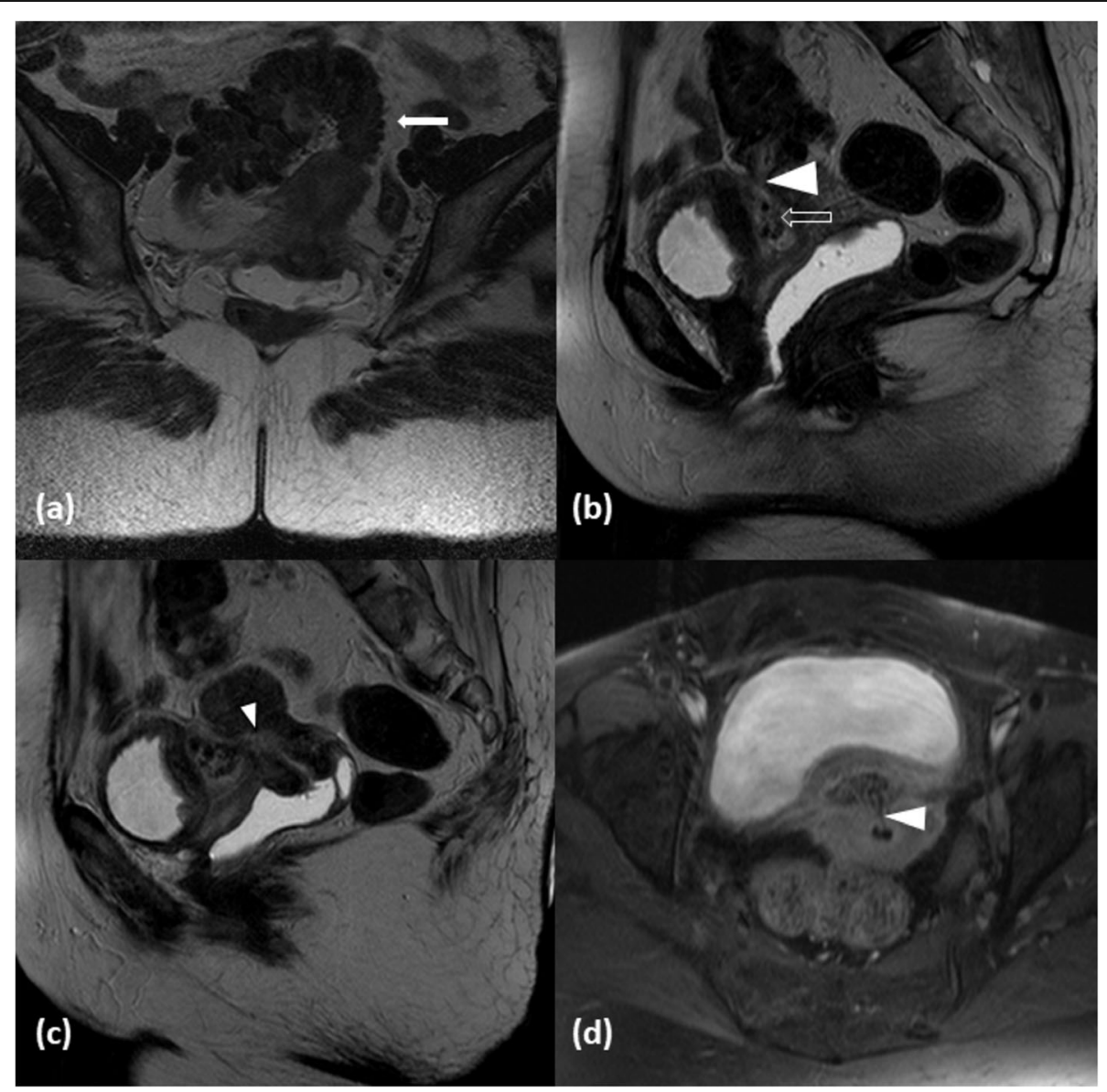

Fig. 2 Magnetic resonance imaging (MRI) of pelvis: T2-weighted axial images (a) shows multiple diverticula arising from sigmoid colon (white solid arrow in a). T2-weighted sagittal images (b and $\mathbf{c}$ ) show a heterogeneous intensity collection representing abscess in the utero-vesical space (void white arrow in $\mathbf{b}$ ) which is arising from the sigmoid colon (white arrowhead in $\mathbf{b}$ ). The fistulous communication (solid white arrowheads in $\mathbf{c}$ and $\mathbf{d}$ ) between the abscess and the uterine cavity is well seen on T2-weighted images (c) and T1-weighed post-contrast axial images (d) 
visualization of pathology along with faster acquisition time of images [9].

Positron emission tomography is an infrequently used modality for the diagnosis. Increased activity in the endometrium is seen in cases of colouterine fistula secondary to carcinoma. However, infection and the inflammatory change associated with diverticulitis can also result in increased standardized uptake values (SUV) [10].

MRI is a non-invasive, detailed, and accurate diagnostic modality with better soft tissue resolution as compared to computed tomography. MRI can delineate the fistulous tract. T1-weighted images are useful in assessing the extension of fistula relative to the adjacent organ and associated inflammatory changes in the surrounding fat planes. Abscesses and collections are better depicted on T2-weighted images and diffusion-weighted image [11].

Surgery is the definitive management of colouterine fistula in most of the cases. In cases with underlying malignancy as the etiological factor of colouterine fistula, en bloc resection is mandatory. For underlying benign conditions, the need for a hysterectomy has not been established. In these cases, resection of the colon and drainage of the purulent uterine lesion is generally adequate as definitive treatment [7].

In cases of colouterine fistula secondary to diverticulitis, a two-stage operative procedure is generally preferred. The first stage involves resection and formation of an end colostomy. This is followed by bowel restoration by reanastomosis of the colon. Percutaneous drainage of intraabdominal abscesses is useful in pre-operative confirmation of diagnosis. It is used for stabilization of the patients and also provides definitive therapy [12].

\section{Conclusion}

Colouterine fistula secondary to sigmoid diverticulitis is a rare complication. CT and magnetic resonance imaging (MRI) play a key role in its diagnosis, preoperative surgical planning, and also in follow-up of these patients. The surgical approach is selected depending on the imaging findings and the condition of the patient.

\section{Abbreviations \\ CT: Computed tomography; MRI: Magnetic resonance imaging; T1WI: T1- weighted imaging; T2WI: T2-weighted imaging; DWI: Diffusion-weighted imaging; CECT: Contrast-enhanced computed tomography; \\ SUV: Standardized uptake value}

\section{Acknowledgements}

We would like to thank Dr. Samaresh Sahu for his support in conducting this study.

\section{Authors' contributions}

SM conceptualized the design of this case report. SM and SP contributed to the acquisition and analysis of patient data and images. UR and VM contributed to the drafting and revision of the case report. DSG contributed to the interpretation of images. All authors have agreed both to be personally accountable for their own contributions, and they have ensured that questions related to the accuracy or integrity of any part of this work, even ones in which they were not personally involved, were appropriately investigated and resolved, and the resolution was documented in the literature. All authors have read and approved the manuscript.

\section{Funding}

No one was paid during this study. The study did not have a source of funding. This study was not supported by a grant.

\section{Availability of data and materials}

All data is available based on a reasonable request.

\section{Ethics approval and consent to participate}

The present study was approved by the ethical board of the hospital in which the study was performed. The patient reported in this article had signed a written informed consent form. This case report was a reporting of a case in a medical educational center, in which all patients are informed that they may be subjects of scientific experiments and are informed of the ethical codes of conducts. This study was in compliance to the latest version of the Helsinki Declaration.

\section{Consent for publication}

The patient had written and signed an informed consent note that the findings may be published without any personal detail.

\section{Competing interests}

The authors declare that they have no competing interests.

\section{Author details}

'Department of Radiodiagnosis and Imaging, Armed Forces Medical College, Pune, India. ${ }^{2}$ Department of Ophthalmology, Armed Forces Medical College, Pune, India.

Received: 25 June 2020 Accepted: 23 August 2020

Published online: 01 September 2020

\section{References}

1. Murphy T, Hunt RH, Fried M, Krabshuis JH (2007). World Gastroenterology Organisation practice guidelines: diverticular disease. World Gastroenterology Organisation. Available via https://www. worldgastroenterology.org/guidelines/global-guidelines/diverticular-disease/ diverticular-disease-english. Accessed 14 Aug 2020.

2. Vilallonga R, Baena JA, Fort JM, Gonzalez O, Gemar E, Armengol Carrasco M (2008) Colouterine fistula complicating diverticulitis in elderly women. Int J Color Dis 24(5):599-600. https://doi.org/10.1007/s00384-008-0630-x

3. Nguyen GC (2011) Epidemiological trends and geographic variation in hospital admissions for diverticulitis in the United States. World J Gastroenterol 17(12):1600. https://doi.org/10.3748/wjg.v17.112.1600

4. Goenka MK, Nagi B, Kochhar R, Bhasin DK, Singh A, Mehta SK (1994) Colonic diverticulosis in India: the changing scene. Indian J Gastroenterol 13(3):86-88

5. Yu NC, Raman SS, Patel M, Barbaric Z (2004) Fistulas of the genitourinary tract: a radiologic review. RadioGraphics 24(5):1331-1352. https://doi.org/10. $1148 /$ rg. 245035219

6. Jemtel L (1909) Des fistulas intestino-uterines. Arch Prov Chir 18:628-654

7. Choi PW (2012) Colouterine fistula caused by diverticulitis of the sigmoid colon. J Korean Soc Coloproctol 28(6):321-324. https://doi.org/10.3393/jksc. 2012.28.6.321

8. Takada T, Nakagawa S, Hashimoto K, Sone K, Kugu K, Kozuma S, Taketani Y (2004) Preoperative diagnosis of colouterine fistula secondary to diverticulitis by sonohysterography with contrast medium. Ultrasound Obstet Gynecol 24(6):682-683. https://doi.org/10.1002/uog.1706

9. Beattie GC, Nelson M, McMillen IM, McMurray AH (2005) Colouterine fistula mimicking pyometrium--diagnosis established with multi-detector computed tomography. Ulster Med J 74(1):51-53

10. Blake MA, Singh A, Setty BN, Slattery J, Kalra M, Maher MM, Sahani DV, Fischman AJ, Mueller PR (2006) Pearls and pitfalls in interpretation of 
abdominal and pelvic PET-CT. Radiographics 26(5):1335-1353. https://doi. org/10.1148/rg.265055208

11. Kassab A, El-Bialy G, Hashesh H, Callen P (2008) Magnetic resonance imaging and hysteroscopy to diagnose colo-uterine fistula: a rare complication of diverticulitis. J Obstet Gynaecol Res 34(1):117-120. https:// doi.org/10.1111/j.1447-0756.2007.00713.x

12. Meadows JM (2019) Acute diverticulitis: imaging and percutaneous drainage. in multidisciplinary approaches to common surgical problems. Springer, Cham, pp 207-216

\section{Publisher's Note}

Springer Nature remains neutral with regard to jurisdictional claims in published maps and institutional affiliations.

Submit your manuscript to a SpringerOpen ${ }^{\mathcal{O}}$ journal and benefit from:

- Convenient online submission

- Rigorous peer review

- Open access: articles freely available online

High visibility within the field

- Retaining the copyright to your article

Submit your next manuscript at $\boldsymbol{\sim}$ springeropen.com 